crème conservée, et aussitôt que celle-ci était disponible. D'après l'examen chimique, la fonction vitaminique avait diminué jusqu'à environ trois quarts de la valeur initiale. Egalement, de la figure 5 , il semble résulter quelque détérioration, ce qui apparaît le mieux dans les courbes de croissance relatives à 50 et à $121 / 2$ milligrammes de matière grasse de beurre. Dans cette expérience aussi il y a done concordance, bien que, par les essais biologiques, on pût conclure moins nettement à une détérioration, que par les dosages chimiques, ce qui, vraisemblablement, est dû aux causes d'erreurs signalées plus haut.

\title{
Résumé
}

Au cours de neuf expériences, nous avons examiné, au moyen de méthodes chimico-physiologiques, la tenue de la vitamine $\mathrm{A}$ et du carotène dans la conservation du lait et de la crème d'après le procédé Hofius. Dans cinq de ces expériences, nous avons également effectué des essais biologiques sur des rats. L'excès de pression d'oxygène pendant la conservation était dans la plupart des cas de 10 atmosphères, la température de 4 à $5^{\circ} \mathrm{C}$, et la durée de la conservation de un à trois mois.

De l'examen chimique, il résultait qu'après environ deux mois de conservation, le carotène aussi bien que la vitamine $\mathrm{A}$ restait intact en majeure partie; des essais biologiques, il ne résultait pas davantage de diminution notable de la fonction "vitamine A " totale. Quelquefois, dans ces recherches chimiques et biologiques, la détérioration était minima, en d'autres cas elle était un peu plus grande ; dans toutes les expériences, plus des deux tiers des quantités initiales de "carotène + vitamine $A$ " furent retrouvés après la conservation.

Il a semblé que, dans des récipients en cuivre étamé, la perte était un peu plus grande que dans des récipients en acier inoxydable.

\section{CONSIDÉRATIONS SUR LA MISE EN BOUTEILLES STÉRILE DU LAIT}

\author{
par \\ E. HAUCK \\ Docteur-Ingénieur.
}

Aujourd'hui, même dans les meilleures exploitations laitières, on ne peut assurer au lait en bouteilles, maintenu à la température des locaux en été, une conservation supérieure à 24 ou 36 heures. C'est par millions que se chiffrent les pertes de lait dans chaque pays. (D'après les statistiques du Ministère de l'Agriculture allemand 
les pertes de lait en Allemagne sont évaluées à 700 millions de francs; en France, elles doivent être à peine inférieures à cette somme, et ce chiffre eomprend surtout la majeure partie des pertes de lait frais.)

Les méthodes, pour conserver le lait frais sans altérer ses qualités, sont anciennes, elles n'ont donné pratiquement jusqu'à ces derniers temps aucun résultat, de sorte que les producteurs de lait se sont résignés à supporter leurs pertes. Et cependant, il n'est pas impossible d'obtenir la conservation du lait en bouteilles. Depuis une dizaine d'années, on est parvenu en Hollande, en Belgique, etc., à conserver le lait destiné à l'approvisionnement des navires et à l'exportation dans les colonies. Le lait est mis en bouteilles de la manière habituelle dans des bouteilles propres mais non complètement stériles, puis soumis dans des autoclaves à des températures de 115 à $150^{\circ}$, ce qui assure en même temps la stérilisation dẹs bouteilles et de leur contenu. On obtient ainsi du lait qui se conserve pratiquement bien, mais cette conservation revient très cher, et, en outre :

Les éléments caractéristiques du lait sont détruits.

Il apparaît un goût de euisson très prononcé.

Le lait prend une couleur brune et se caramélise en parties.

Peu à peu, le procédé a été perfectionné et, à la suite d'essais ayant duré plusieurs années et d'expériences nombreuses, on est arrivé à obtenir une certaine amélioration du goût et de la qualité du lait ainsi traité sans toutefois parvenir à ce que ce lait stérilisé se rapproche du lait brut ou pasteurisé. Par exemple, à la place des autoclaves, on a employé des stérilisateurs rotatifs de bouteilles dans lesquels le lait est mis en mouvement, ce qui a permis de réduire la durée de la stérilisation du lait.

Il est cependant reconnu que le problème de la stérilisation du lait en bouteilles et des bouteilles elles-mêmes ne peut être résolu de cette manière, ear il n'est pas possible de régler l'action de la chaleur de façon à détruire tous les germes et spores sans occasionner de ce fait une modification des qualités essentielles du lait.

Il est apparu clairement aux praticiens qu'il était nécessaire de séparer la question de la stérilisation du lait de celle des bouteilles. Après des premiers essais de soutirage de lait stérile dans des bouteilles remplies d'un gaz inerte (oxygène, etc.) qui n'ont pas donné de résultat pratique, on s'est tourné vers le procédé de la stérilisation des bouteilles et du soutirage stérile qui a fait ses preuves dans d'autres industries, par exemple dans celles des jus de fruits, de la bière, etc. On réussit parfaitement à stériliser et à mettre stérilement en bouteilles des jus de fruits qui sont des liquides beaucoup 
plus délicats que le lait et à leur assurer une conservation de plusieurs années. Les Seitz-Werke, de Bad-Kreuznach et Paris, précurseurs et spécialistes de la question du soutirage stérile dans l'industrie des boissons, ont reconnu de bonne heure l'importance de l'application de cette méthode au lait.

Après des recherches de laboratoire, on a entrepris des essais pratiques dans des exploitations laitières. Par exemple, on a installé dans une des plus importantes laiteries d'Europe Centrale : la laiterie bavaroise à Nuremberg, une machine à laver les bouteilles qui assure, non seulement la stérilisation pratique des bouteilles, mais garantit à la sortie de la machine la destruction totale de tous les germes contenus dans les bouteilles.

Les essais vérifiés par les laboratoires particulièrement bien aménagés de cette entreprise ont montré que le but recherché était atteint d'une manière absolue. Les essais préalables pour le soutirage stérile du lait furent entrepris dans la laiterie modèle bien connue de Saarbruck.

Basés sur le principe appliqué dans d'autres branches de l'industrie des boissons, ces essais ont obtenu un succès complet. La question la plus délicate du problème, c'est-à-dire, la stérilisation absolue des bouteilles, la mise en bouteilles et le bouchage stérile, était ainsi résolue. Il était donc possible de soutirer le lait sans que sa teneur en germes augmente entre l'entrée dans la tireuse et la mise en bouteilles.

Pour mettre au point définitivement le procédé dans la pratique, il était seulement nécessaire d'alimenter la soutireuse avec du lait stérile. Le problème de la stérilisation du lait fut justement résolu à peu près à la même époque. A la suite de travaux de spécialistes hollandais, avec le $\mathrm{D}^{\mathrm{r}}$ CARP, d'Amsterdam, on a découvert un procédé de stérilisation du lait et de ses dérivés qui permet d'obtenir des produits absolument stériles.

Dans ce procédé de stérilisation, le lait est porté rapidement et sous pression à une température élevée, puis, aussitôt après, refroidi à la température de soutirage.

Le système Carp, en liaison avec une installation Seitz de rinçage et de soutirage stérile, est appliqué actuellement dans une entreprise laitière de Rotterdam, où, depuis plus d'un an, il a donné les meilleurs résultats.

Le lait stérile ainsi que ses dérivés sont emportés par des navires dans toutes les parties du monde et leur eonservation dure de 5 à 6 mois.

Dans cette installation, le lait ou les produits dérivés du lait sont d'abord débarrassés de leurs impuretés par filtration ou centrifugation, puis réchauffés préalablement, homogénéisés partiel- 
lement et ensuite traités dans le stérilisateur Carp. Ils sont alors conduits dans un tank intermédiaire de régulation et soutirés en bouteilles stérilisées. La machine à laver les bouteilles est du modèle de celle décrite dans la revue "Le Lait» de janvier 1939, pages 6 et 7 . Elle comporte cependant une zone stérilisante particulière. Le rinçage final des bouteilles s'effectue avec de l'eau stérile, et en outre, le dispositif de déchargement et le transporteur d'évacuation sont protégés par un couvercle.

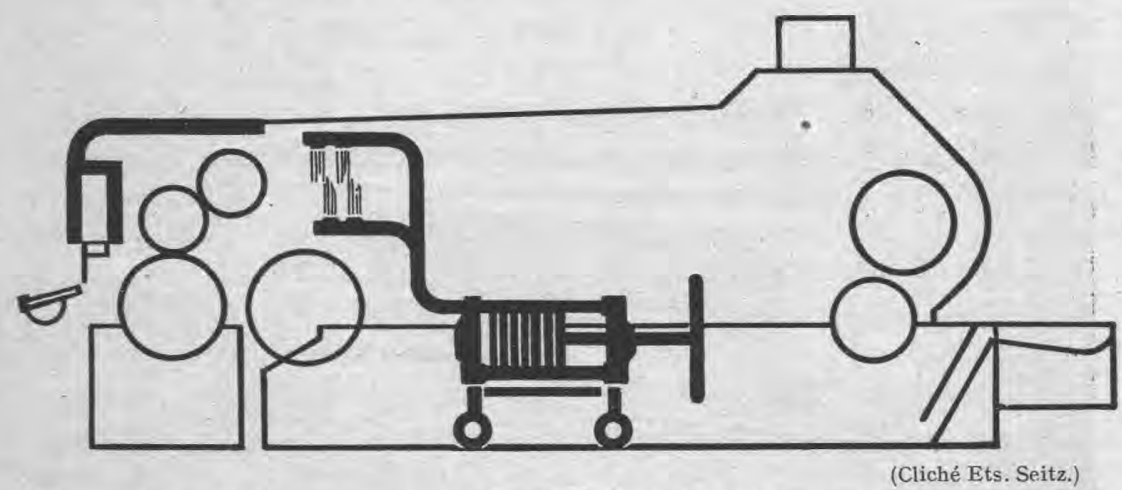

Figure I. - Schéma montrant le dispositif de stérilisation des bouteilles.

Il nous semble intéressant de décrire en quelques lignes le mode de travail d'une installation de soutirage stérile de ce genre.

Les easiers contenant les bouteilles sales sont amenés par un transporteur à rouleaux devant le poste de chargement de la machine automatique sans chaîne ni brosse à laver et stériliser les bouteillés. Celles-ci sont alors enlevées des casiers, placées sur la table de chargement et introduites automatiquement dans les paniers de la machine à grand rendement.

Dans les machines à débit moyen, les bouteilles sont introduites à la main directement dans les alvéoles.

Puis elles sont soumises d'abord à un prétrempage (pour les grands rendements seulement), suivi d'un trempage prolongé dans la lessive et d'injections de lessive surchauffée sous forte pression, ce qui les débarrasse entièrement de leurs impuretés.

Ce trempage de longue durée ainsi que les injections constituent le traitement préliminaire indispensable pour la stérilisation des bouteilles qui est obtenue à l'aide d'eau bouillante additionnée au besoin d'un produit de désinfection.

Avant de quitter la machine, les bouteilles propres reçoivent un dernier rinçage à l'eau stérile. Cette eau est passée auparavant dans un filtre stérilisateur et a été réchauffée par la vapeur à la tempéra- 
ture voulue. Les bouteilles ainsi nettoyées et stérilisées sont déchargées sur un transporteur couvert et conduites automatiquement à la machine à soutirer et boucher. Les transporteurs de déchargement et d'évacuation sont protégés par une sorte de

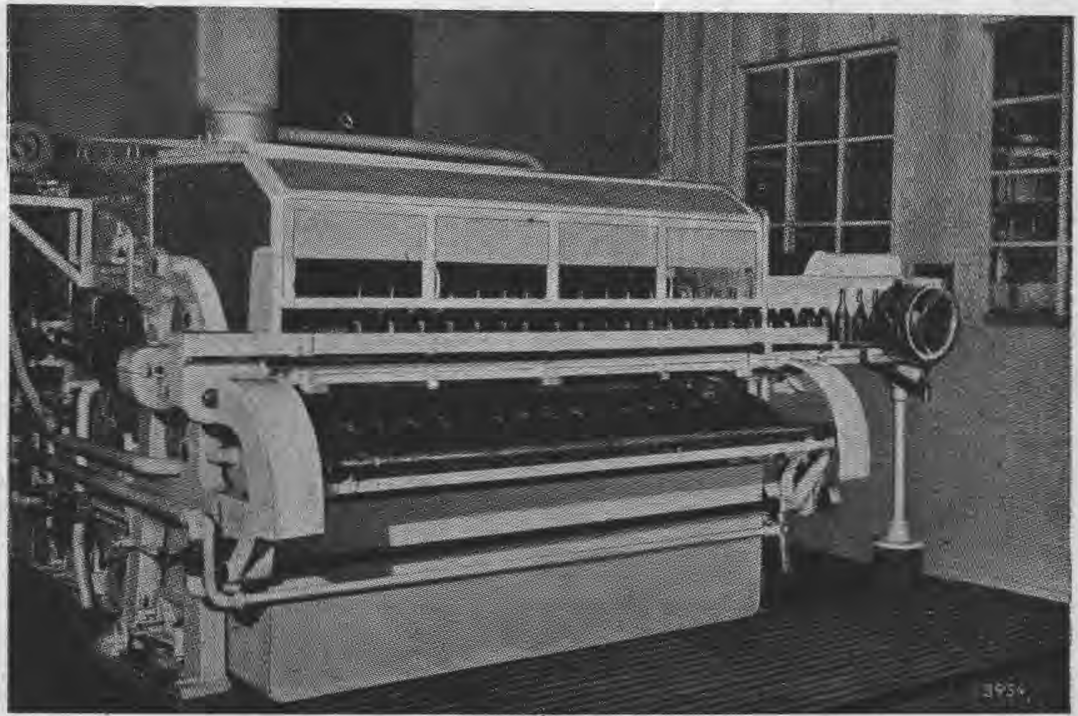

(Cliché Ets. Seitz.)

Figure 2. - Machine à grand rendement pour le nettoyage et la stérilisation des bouteilles.

tunnel. Ils sont constamment remplis d'air stérile surpressé de même que les postes de soutirage et de bouchage également recouverts. De cette manière, on évite les risques d'infection des bouteilles ou du produit par contact avec l'air extérieur non stérile.

La soutireuse stérile est du modèle des tireuses à différence de pression. Les bouteilles quittent automatiquement le transporteur par une étoile d'entrée, elles sont ensuite appliquées sur les robinets de remplissage, soit par des pistons à air comprimé, soit par une courbe de montée. La pression dans le réservoir est maintenue par de l'air stérile. Le lait qui peut déborder des bouteilles après le remplissage est recueilli et entraîné à l'extérieur. On empêche ainsi les risques d'infection du produit et des bouteilles.

Les bouteilles remplies sont ramenées automatiquement par l'étoile de sortie sur le transporteur et conduites à la machine à boucher. Cette machine est prévue pour le bouchage métallique : capsules aluminium, couronne ou autres. Les fermetures sont d'abord stérilisées, elles arrivent automatiquement dans le canal 
d'amenée et sont serties automatiquement sur les goulots des bouteilles. Par mesure de sécurité, elles sont encore flambées avant d'être serties.

Toute l'installation de remplissage et de bouchage est protégée par un earter rempli d'air stérile surpressé.

Les bouteilles emplies et bouchées quittent alors l'installation stérile, on les enlève de la table d'accumulation et on les place en casiers. A ce moment elles sont prêtes à être expédiées, leur conservation durera des semaines et des mois et elles pourront être en tout temps servies au consommateur. Le mode de chauffage du lait qui

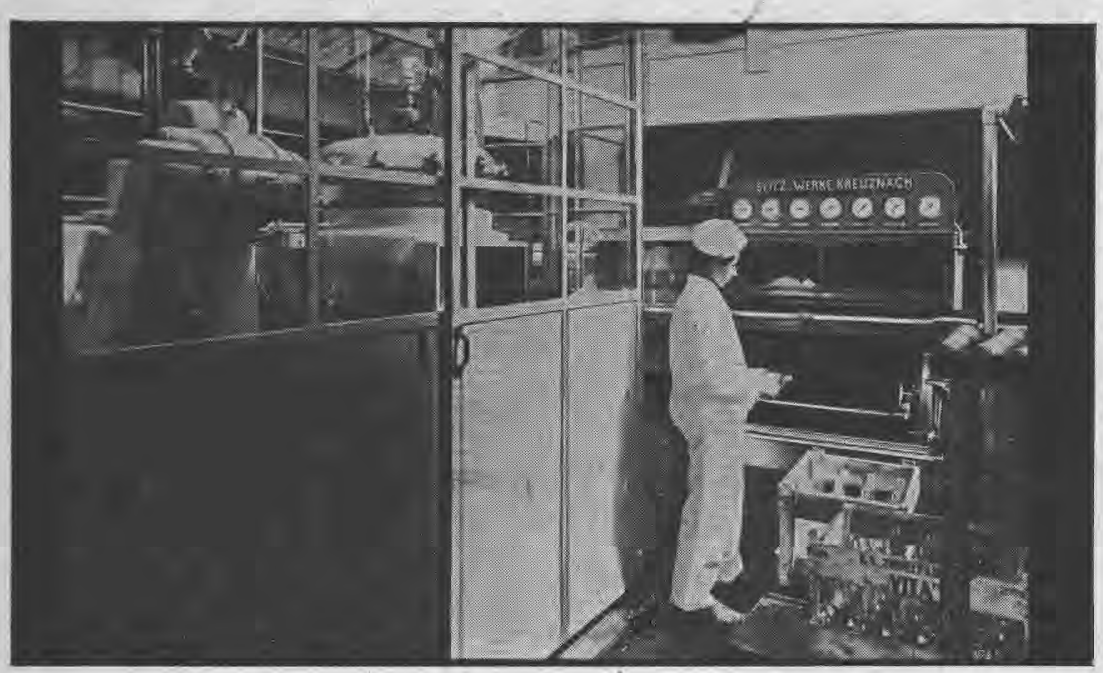

(Cliché Ets. Seitz.)

Figure 3, - Installation moyenne de rinçage et de stérilisation.

applique les nouvelles méthodes de traitement à haute température supprime les inconvénients de l'action prolongée de la ehaleur qui nuit aux qualités du lait. Cette action ne dure que juste le court espace de temps indispensable pour la destruction des germes et des spores du lait brut. Ainsi la saveur, la couleur et la composition initiale du lait sont conservées dans le lait stérilisé. Ce lait est mis en bouteilles stérilement sans aucune modification de ses qualités.

Dans le procédé décrit ci-dessus, les opérations de nettoyage et de stérilisation des bouteilles sont réunies sur la même machine qui fonctionne en liaison directe avec la soutireuse et la boucheuse. Des essais pratiques ont montré qu'il était également possible de séparer le nettoyage de la stérilisation et d'entreprendre ces travaux à l'aide d'appareils indépendants. Toutefois, il est nécessaire que la machine à laver ne procure pas seulement des bouteilles 
propres, mais il faut encore que celles-ci aient subi un commencement de stérilisation, c'est-à-dire, qu'elles aient été soumises à l'action suffisamment prolongée de la lessive à haute température. Vient ensuite l'inštallation de stérilisation reliée directement à la laveuse. Le stérilisateur sera par exemple du modèle qui a fait ses preuves pour la stérilisation des bouteilles de jus de fruits. Après avoir été

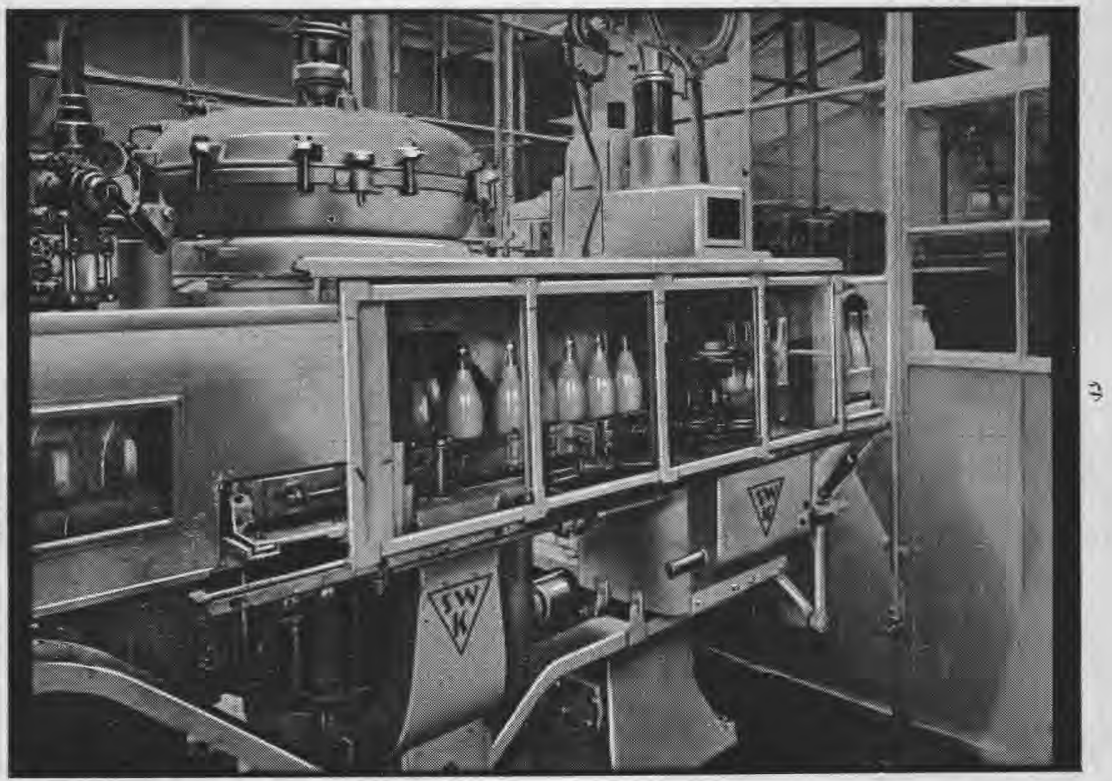

(Cliché Ets. Seitz.)

Figure 4. - Machines à emplir et à boucher pour soutirage stérile.

stérilisées sur cet appareil, les bouteilles seront conduites automatiquement vers la soutireuse stérile décrite précédemment.

Dans un but de simplification du travail, on recommande pour les installations nouvelles de prévoir la machine unique de nettoyage et de stérilisation. Par contre, dans les installations existantes, le deuxième système est préférable, e'est-à-dire, que d'un côté, on utilisera l'installation de rinçage et de soutirage normale qui sera complétée de l'autre côté par un stérilisateur de bouteilles et une soutireuse stérile. Ainsi on aura la faculté de soutirer normalement d'une part le lait et ses produits dérivés et de faire d'autre part la mise en bouteilles stérile.

Les possibilités d'application du procédé sont très nombreuses. On peut aujourd'hui préparer du lait au chocolat, produit essentiellement périssable, qu'il est possible maintenant de conserver et de 
vendre pendant plusieurs mois au même titre que les limonades ou les jus de fruits et que l'on peut ainsi stocker.

Il en est de même pour les crèmes et les laits condensés que l'on peut conserver dans des récipients en verre. Pour le lait de consommation journalière, les applications du procédé sont encore limitées. Néanmoins, il est prou vé que l'emploi du soutirage stérile, par une simplification du procédé, - des essais pratiques sont actuellement en cours - permet de prolonger sensiblement la conservation du lait en bouteilles en général tout en lui maintenant ses qualités. On peut atteindre aujourd'hui une conservation de 3 à 4 jours.

Il faut espérer que dans un avenir proche des essais seront entrepris pour résoudre en détail le problème de l'aug mentation de la durée de conservation, indépendamment de la stérilisation absolue et du soutirage stérile.

Cette question ouvrira sans aucun doute de nombreux débouchés à l'industrie laitière. Que l'on réussise tout d'abord à produire du lait en bouteilles se différenciant à peine du lait pasteurisé et ayant une durée de conservation de plusieurs jours, il est incontestable que ceci aura naturellement une influence sur le mode de travail des entreprises et sur le prix de revient, non seulement, en raison du fait que le consommateur recevra son lait en bouteilles sous une forme meilleure qui, grâce à la conservation, évitera les pertes, mais aussi parce qu'on pourra ainsi réduire les frais de l'entreprise, ce qui aura pour résultat final d'ouvrir de nouveaux débouchés à l'industrie du lait en bouteilles présenté de cette manière essentiellement hygiénique.

\section{SÉPARATION DU LACTOSE ET DES PROTÉINES SOLUBLES CONTENUES DANS LE SÉRUM DU LAIT PAR EXTRACTION PAR L'ALCOOL}

par G. GÉNIN

Ingénieur E. P. C.

On sait déjà depuis longtemps, à la suite d'études expérimentales entreprises sur la question, que la poudre de petit-lait ou de sérum provenant de la fabrication de la caséine peut être extraite au moyen de solutions alcooliques. On a constaté, en opérant avec des solutions alcooliques de différentes concentrations, qu'il existe, au voisinage d'une concentration en alcool de $70,7 \%$, une zone étroite au-dessus de laquelle la précipitation par l'alcool des constituants de la poudre n'entraîne pas de modification, de la solubilité des protéines du sérum dans l'eau, mais au-dessous de laquelle cette solubilité est diminuée. 